\title{
The research on Breaker Fault Status Parameter Classification of Improved Particle Swarm Optimization
}

\author{
Yihang Sun \\ Beijing Institute of Mechanical Equipment, Beijing, China 100854)
}

\begin{abstract}
In order to improve the mechanical structure of the type of fault resolution precision high voltage circuit breaker spring mechanism, the paper analyzes the characteristics of the circuit breaker and the combination of mechanical vibration signal PSO algorithm (PSO) SVM parameter optimization method proposed collaborative dynamic acceleration constant inertia weight particle swarm optimization (WCPSO) optimization support vector machine (SVM) analysis breaker fault classification parameters and kernel function parameters. The vibration signal circuit breaker empirical mode decomposition, the total intrinsic mode components through energy analysis to obtain the required fault feature vectors and support vector machine as input, the use of dynamic acceleration constant synergy inertia weight PSO support vector machines penalty factor $C$ and radial basis kernel function parameters $\sigma$ optimize the fault feature vector signal input test samples after SVM training sample trained optimized for fault classification, fault status classification. The experimental analysis of this method can effectively improve the resolution of the breaker failure signal type Accuracy.
\end{abstract}

Keywords -circuit breaker; SVM; vibration signal; PSO; energy method; fault diagnosis

\section{INTRODUCTION}

With the maturity distribution automation systems, circuit breakers and distribution system as an important device has important significance for its fault diagnosis. Mechanical vibration signals generated breakers in sub-closing action contains a lot of state information, has important practical implications for the analysis of vibration signals of these [1].

These frontier of contemporary theories and methods will inevitably penetrate into the diagnostic techniques in the past, making the diagnosis can almost simultaneous development of these frontier[2-3]. Fault diagnosis expert system, neural network as an important branch of artificial intelligence, attention and has been widely applied. Swarm intelligence optimization technology as a new technology, is a new hot field of artificial intelligence research, which will be used in PSO breaker fault diagnosis, a new hotspot[4-7].

Since the 1990s, swarm intelligence (Swarm Intelligence) research has aroused great attention of many scholars, social insects swarm intelligence is inspired by a series of new solutions to complex problems through traditional simulation generated by their actions, it is the simple dumb many groups of individuals by a simple mutual cooperation between the intelligent behavior exhibited. Among them, the PSO[8] (Particle Swarm Optimization, PSO) is a J.Kennedy and RCEberhart proposed in 1995 based on the principle of swarm intelligence optimization algorithms, foraging birds originated from the process of migration and aggregation simulation. It converges fast, easy to implement and only a few parameters need to be adjusted, and thus was put forth to become intelligent optimization and evolutionary computing a new hotspot[9-11].

Reflected throughout the particle swarm particle in search of food sources (optimal solution) in both maintain their own inertia, and the use of individual cognition and social cognition continue to modify their flight direction, eventually leading towards a food source close to the group. Of course, there are easy-to-particle swarm into local optimum result in low convergence accuracy and convergence easily shortcomings[12].Particle Swarm Optimization (PSO) algorithm as a group of intelligent creatures by nature predatory behavior simulation and proposed a global search strategy based on the speed and simplicity of the population - the displacement mode, to avoid the complexity of genetic manipulation. Because of its calculation speed and ease of implementation of the algorithm itself, attracted wide attention and research on a number of international scholars[13].

According to relevant literature and research trends about love PSO algorithm in the field of evolutionary algorithm analysis, currently there are several research direction[5, 14].

How to use effective mathematical tool for runtime behavior of PSO, convergence, convergence speed, parameter selection, parameter robustness and computational complexity of the analysis should be the current research focus[15]

\section{SVM PARAMETERS AND THEIR KERNEL FUNCTION PARAMETERS}

Support vector machines can be divided into two-dimensional space as shown in Fig(1), rectangular and oval dot points represent two types of data samples, $\mathrm{H}$ is the sorting line, $\mathrm{H} 0$ and $\mathrm{H} 1$ represent samples classified 
by line and parallel to the nearest sample classification line spacing interval between them became classification. When the total sample linear separability exists a hyperplane asked two types of training samples can be completely separated from the sample points on $\mathrm{H} 0$ and $\mathrm{H} 1$ is the support vector.

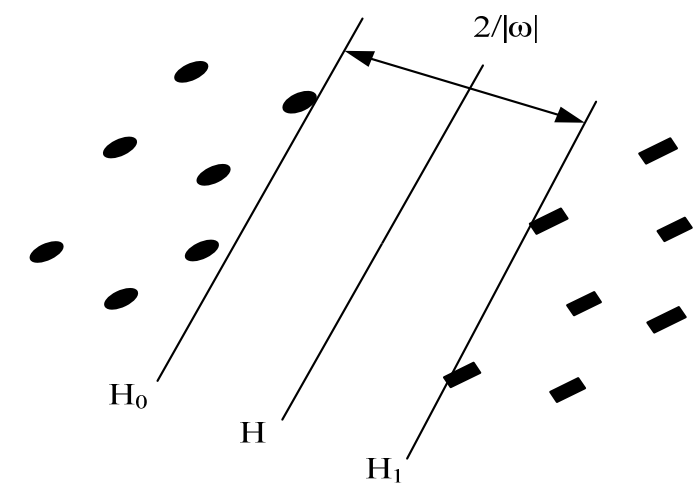

FIGURE I CLASSIFICATION LINE BASED ON 2-D LINEAR SEPARABILITY

The actual situation in most cases of non-linear, in this case, SVM generally taken to introduce slack variables $\xi_{i}$ and penalty factor $\mathrm{C}$ form hyperplane constraints:

$$
y_{i}\left[\left(\omega \cdot x_{i}+b\right)\right] \geq 1-\xi_{i} \quad \mathrm{I}=1,2, \ldots \mathrm{N}
$$

Where $\omega$ is the surface normal vector classification, $b$ is the classification threshold. The introduction of the generalized problem of optimal classification face Lagrange multipliers, that is to build the generalized optimal hyperplane problem can be converted to solve the dual quadratic programming problems, the introduction of kernel function in SVM classification function, namely:

$$
f(x)=\operatorname{sgn}\left\{\left(\sum_{i=1}^{n} a_{i}^{*} y_{i} K\left(x_{i}, x\right)+b^{*}\right)\right\}
$$

Where $a_{i}^{*}$ is the Lagrange multiplier, $y_{i}$ is class value, $K\left(x_{i}, x\right)$ is the kernel function, $b^{*}$ is the classification threshold. According to functional theory, as long as the kernel function $K(x, y)$ satisfies Mercer conditions, nonlinear transformation can be achieved. Commonly used kernel functions are mainly polynomial kernel function, radial basis function, Sigmoid kernel function. RBF kernel function is selected for the vibration characteristics of the letter and the feature vector:

$$
K\left(x_{i}, x\right)=e^{\frac{-\left\|x-x_{i}\right\|^{2}}{\sigma^{2}}}
$$

Where $x_{i}$ is the input feature vector, $\sigma$ is the kernel function parameters.

SVM classification algorithm is divided into: "one to many", "one", a one-time solution algorithm, decision directed acyclic graph support vector machines. In this paper, "many" support vector machine classification algorithm.

SVM model classification accuracy with the penalty factor $\mathrm{C}$ and kernel function exist in certain relations, in order to obtain the best classification performance of SVM model, you need to get the best value of $\mathrm{C}$ and kernel function values. Obviously this is an optimization problem, if taken exhaustive way to find the optimal value, very large, can not be achieved.

Particle swarm optimization with implicit parallelism, fast convergence, set parameters and less powerful global search capability, you can search within a very short period of time to the global optimum.

\section{FEATURE Vectors EXTRACted BASED ON the TOtAL WEIGHT OF ENERGY BY IMF}

According to the test signal and the theoretical knowledge known techniques, regardless of the actual dimensions in the case of the oscillating signals $x(t)$ time integral of the square of $x^{2}(t)$ is called the energy of the signal:

$$
Q(i)=\int_{-\infty}^{+\infty}|x(t)|^{2} d t
$$

When the signal is ${ }^{x(t)}$ discrete signal, the signal energy value added and interval signal, namely:

$$
Q(i)=\sum_{t=0}^{n}\left|x^{2}(t)\right| \Delta t
$$

Where $\Delta t$ is the sampling interval, the sampling interval is herein $0.05 \mathrm{~ms}, Q(i)$ is the total energy of the signal on the whole interval segment

IMF energy after a fault signal when the value of the EMD, a failure to obtain $c_{1}(t), c_{2}(t) \ldots c_{n}(t)$ series of major IMF component information contained frequency components contained in the end these IMF components, circuit breakers of different mechanical failure occurs from high closing vibration signal will be generated difference.

Fault classification process block diagram shown in Figure 2, the signal is first collected for EMD decomposition, IMF and extract feature vector component fault signal input in learning good SVM classification.

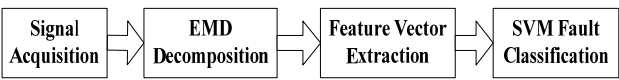

FIGURE II PROCESS OF STATE DETECTION 
(1) The original vibration signals of EMD, take the first n IMF component contains major faults, this paper takes the first six IMF components.

(2) Calculation of each IMF (discrete signal) a total energy component $Q(i)$ :

$$
Q(i)=\sum_{t=0}^{n} c_{i}^{2}(t) d t \quad\left({ }^{n=5000}\right)
$$

(3) The components of the total energy for IMF normalized:

$$
E_{\mathrm{i}}=\frac{Q(i)}{E}
$$

Where $E_{\mathrm{i}}$ is the total energy IMF component, $E$ is the total energy of the original vibration signal.

(4) IMF component to the total energy of the element structure eigenvectors T:

$$
T=\left[E_{1}, E_{2}, \ldots, E_{n}\right]
$$

(5) Collaborative dynamic acceleration constant inertia weight PSO optimized SVM get better classification performance, using the training data SVM training.

(6) The fault input feature vectors of training samples tested in good optimized SVM, given the state of the circuit breaker failure type.

\section{Collaborative Dynamic AcCELERATion CONSTANT INERTIA WEIGHT COEFFICIENT PARTICLE SWARM OPTIMIZATION METHOD}

Particle Swarm Optimization (PSO) is the basic idea of the potential solutions to each problem are particles of the search space, the fitness of all particles have a function to be optimized decisions, each particle has a velocity vector decided they fly direction and distance, and then follow the current optimal particles are particles in the solution space to search. PSO optimization function can control the classification accuracy of classification penalty factor $\mathrm{C}$ and radial basis kernel function parameters $\sigma$, classification function to achieve optimal performance of this method.

Inertia weight factor $\omega$ describes the impact on the current generation of the generation rate of speed on particle size can be adjusted to control the value of global and local search ability of PSO. When $\omega_{\text {is large, the }}$ impact of the previous rate of greater global search capability and strong; $\omega^{\omega}$ is small, less affected by the speed of the previous, relatively strong local search ability. Weight function coefficients by right sizing the local minima. The basic PSO is inertia weight factor is a special case, in order to better control algorithms to detect and development capabilities to introduce inertia weight factor, namely PSO with inertia weight particle swarm optimization (WPSO).

PSO tend to search the optimal solution is composed of two random acceleration constant cognitive factors and social factors c1 c2 guidance, c1 and c2 represent the stochastic particle acceleration value to their extreme right and extreme global advance. If $\mathrm{c} 1=\mathrm{c} 2=0$, then the particles will be flying this speed until the border. In this case, the particles can only search a limited area, it is difficult to find a good solution. When $\mathrm{c} 1=0$, then no cognitive particles in particle interactions, the ability of the new algorithm search space. Its faster convergence rate than the standard algorithm, but encounter complex problems more easily than the standard algorithm into local pole. When c2 $=0$, there is no sharing of information between the community particles, a size of the group is equivalent to running $\mathrm{m}$ a single particle, thereby obtaining a very low probability of the optimal solution.

Elementary particle swarm optimization algorithm for updating the formula:

$$
v_{i}^{k+1}=v_{i}^{k}+c_{1} r_{1}\left(p_{i}^{k}-x_{i}^{k}\right)+c_{2} r_{2}\left(p_{n}^{k}-x_{i}^{k}\right), i=1,2, \ldots
$$

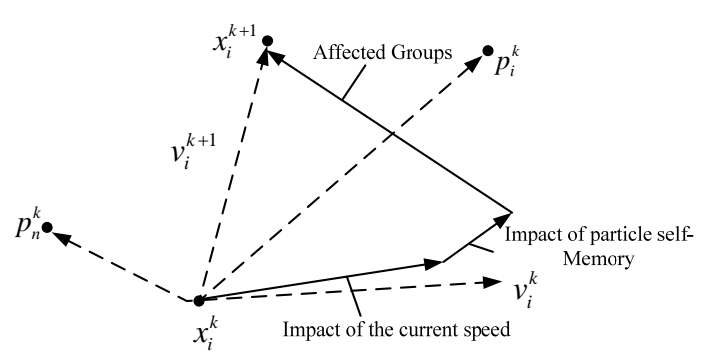

FIGURE III PARTICLE LOCATION UPDATE

Where, ${ }_{i}^{k+1}$ denotes the i-th $(\mathrm{k}+1)$ particle generation during the flight speed; $x_{i}^{k+1}$ i represents a position of the first $k+1$ particle generation; ${ }^{p_{i}^{k}}$ represents the i-th particle up to the first generation of the found $k$ excellent location; $p_{n}^{k}$ represents the current population to the current position to the optimum position to; $p_{i}^{k}-x_{i}^{k} \quad$ represents individual cognition; $p_{n}^{k}-x_{i}^{k}$ represents social cognition; affect $\omega$ inertial coefficient, the previous generation of this generation. 


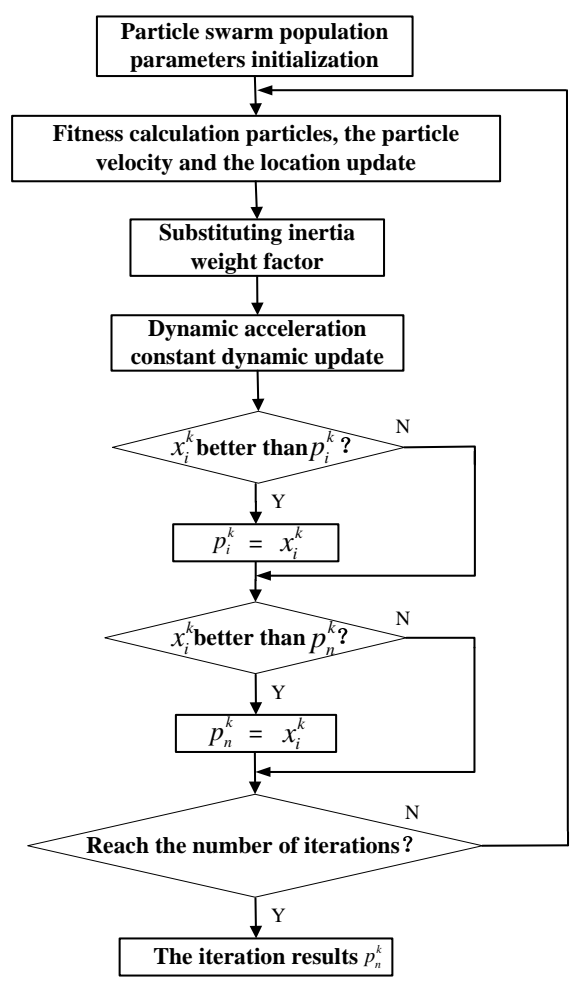

FIGURE IV PARTICLE SWARM OPTIMIZATION PROCESS

Constant optimization of the dynamic equation and particle update formula:

\section{$\mathrm{C} 1=\mathrm{R} 1+\mathrm{R} 2 . \mathrm{T} / \mathrm{TMAX} \quad \mathrm{C} 2=\mathrm{R} 3-\mathrm{R} 4 . \mathrm{T} / \mathrm{TMAX}$}

$\mathrm{R} 1, \mathrm{R} 2, \mathrm{R} 3, \mathrm{R} 4$ as the initial setting. $\mathrm{t}$ is the current update algebra, Tmax is the maximum update algebra. Wherein the selection is to select the initial setting of the simulation function calculated by the algorithm in the optimal range of values.

$$
\begin{gathered}
v_{i}^{k+1}=\omega v_{i}^{k}+c_{1} r_{1}\left(p_{i}^{k}-x_{i}^{k}\right)+c_{2} r_{2}\left(p_{n}^{k}-x_{i}^{k}\right), i, k=1,2, \ldots \\
x_{i}^{k+1}=x_{i}^{k}+v_{i}^{k+1}, \mathrm{I}, \mathrm{K}=1,2, \ldots \mathrm{N}
\end{gathered}
$$

Where $\omega$ is a coefficient of inertia weight, acceleration constants c1, c2 are non-negative constants, r1, r2 uniformly distributed random numbers $0-1$ on.

4 Improved PSO_SVM breaker fault condition in the classification

In this paper, the number of employed population particle swarm is 30 , the maximum is 300 algebra. $\mathrm{R} 1=$ $1.2, \mathrm{R} 2=0.6, \mathrm{R} 3=5, \mathrm{R} 4=2.2$ for the analysis and optimization functions by generalized derived this number is dynamic acceleration constant optimization best initial settings, $\omega=0.8$ (typically 0.4 -values between 1.2 ). Kernel function using radial basis function, Fitness function is the accuracy verify under the cross-classification.optimized SVM penalty factor C and radial basis function $\sigma$, optimization steps are as follows:

(1) the optimization of the parameters $\mathrm{C}$ and $\sigma$, the value range is constraint $C: 0.1-1000, \sigma: 0.01-100$, in the definition of the space 30 are randomly generated initial population of particles: pop $(i, 1)=x 1, x 2, \ldots$, xi and pop $(i, 2)=y 1, y 2, \ldots$, yi. Set the maximum evolution generation Tmax $=300$, the current evolution algebra set $\mathrm{t}$ $=1$, by formula (10) to calculate the initial value of dynamic acceleration constants $\mathrm{c} 1=1.22 \mathrm{c} 2=4.93$, produce changes in the composition of the initial displacement of each particle displacement matrix:

$$
\text { POP(I,J) I=1,2, ., 30; J=1,2 }
$$

(2) the use of the fitness function, namely to verify the accuracy of cross-classified under the calculated fitness value of each particle.

(3) compare the fitness value of particles and their optimal values. If the current value is better than their own, then set to the current value.

(4) comparing the value of the particle populations to adapt to the optimal value. If the current value is better than the population, the value of the current set of particles.

(5) according to formula (11) and (12) of particle velocity and a location update.

(6) Check the termination condition, if not satisfied algebra Tmax $=300$, then $t=t+1$ Go (2). End conditions for optimizing the maximum evolution generation $\mathrm{Tmax}=$ 300.

Optimization results obtained by SVM classification carried out in the breaker failure status classification, and then compare optimized.

\section{EXPERIMENTAL RESULTS AND ANALYSIS}

WCPSO-SVM using particle swarm optimization algorithm for support vector machine parameters and kernel function parameters were optimized.

30 sets of test data for each failure, each of 20 randomly selected as the training data set SVM training, and the remaining 10 groups each test as the test data analysis. Results of the test results takes 10 , as shown in Tab1. 
TABLE I WCPSO PARAMETER OPTIMIZATION RESULTS

\begin{tabular}{cccc}
\hline WCPSO & $C$ & $\sigma$ & $\begin{array}{c}\text { Classification } \\
\text { accuracy(\%) }\end{array}$ \\
\hline 1 & 278 & 2 & 82.5 \\
2 & 78 & 0.9 & 92.5 \\
3 & 78.1 & 85.3 & 85 \\
4 & 54.8 & 0.9 & 97.5 \\
5 & 49.2 & 32 & 87.5 \\
6 & 12.6 & 6 & 92.5 \\
7 & 27.9 & 65 & 90 \\
8 & 569 & 33.6 & 92.5 \\
9 & 113 & 21.9 & 77.5 \\
10 & 27 & 47.3 & 75
\end{tabular}

As can be seen from the table when the SVM penalty factor $\mathrm{C}=54.8$ and radial basis function $\sigma=0.9$, the performance out of the classification accuracy, highest performance.

In this paper, "many" support vector machine classification algorithm and IMF component of total energy method and the combination of EMD breaker vibration signal extracted feature vectors.

In order to verify the advantages WCPSO this optimization method, firstly no parameter optimization of SVM, PSO parameter optimization of SVM and SVM parameter optimization WCPSO the training and test data were analyzed. SVM uses "many" classification thinking, as opposed to other methods, it can better control the classification accuracy. As Tab2 shows, Time refers to the classification table from iteration to optimize the completion time classification,for optimizing the classification function without taking 100 penalty factor C and radial basis function parameter $\sigma$ optimization results take 2; classification function for PSO take 36.9 penalty factor $\mathrm{C}$ and radial basis function parameter $\sigma$ takes 0.9 optimization results; for WCPSO classification function takes the penalty factor C 54.8 and optimization results RBF kernel function parameter $\sigma$ takes 1.4. Time refers to the classification table from iteration to optimize the completion time classification.

First, we classify the average accuracy rate of 20 times as criteria, so you can optimize the generalization reflects the conclusions. Average accuracy rate can be seen with high accuracy SVM has optimized random values when the average results are not optimized, the average accuracy rate WCPSO optimized in PSO optimization.

Second, and then to optimize the classification of 20 times the average time for comparison, after the lapse of the SVM WCPSO optimized SVM classification data showing the time of the advantages of short computation time.

TABLE II CLASSIFICATION PARAMETERS COMPARE

\begin{tabular}{cccc}
\hline $\begin{array}{c}\text { Parameters to be } \\
\text { optimized }\end{array}$ & $\begin{array}{c}\text { No } \\
\text { optimization }\end{array}$ & PSO & WCPSO \\
\hline$C$ & 100 & 36.9 & 54.8 \\
$\sigma$ & 2 & 0.9 & 1.4 \\
$\begin{array}{c}\text { Classification } \\
\text { accuracy(\%) } \\
\begin{array}{c}\text { Classification } \\
\text { time(s) }\end{array}\end{array}$ & 78.1 & 85.3 & 92.6 \\
\hline
\end{tabular}

TABLE III OPTIMIZATION PARAMETERS CLASSIFICATION RESULTS

\begin{tabular}{|c|c|c|c|c|c|c|c|c|c|}
\hline $\begin{array}{c}\text { SVM } \\
\text { parameter } \\
\text { optimization } \\
\text { algorithm } \\
\text { contrast } \\
\end{array}$ & $\begin{array}{c}\text { The } \\
\text { particle } \\
\text { population }\end{array}$ & $\begin{array}{c}\text { The } \\
\text { initial } \\
\text { setting } \\
\text { value } \\
R_{1} \\
\end{array}$ & $\begin{array}{c}\text { The } \\
\text { initial } \\
\text { setting } \\
\text { value } \\
R_{2} \\
\end{array}$ & $\begin{array}{c}\text { The } \\
\text { initial } \\
\text { setting } \\
\text { value } \\
R_{3} \\
\end{array}$ & $\begin{array}{c}\text { The } \\
\text { initial } \\
\text { setting } \\
\text { value } \\
R_{4} \\
\end{array}$ & $\begin{array}{c}\text { Inertia } \\
\text { weight } \\
\text { factor } \\
\omega\end{array}$ & $\begin{array}{c}\text { Acceleration } \\
\text { constant } \\
C_{1} 、 c_{2}\end{array}$ & $\begin{array}{c}40 \text { sets of } \\
\text { data/the } \\
\text { average } \\
\text { classification } \\
\text { of } 20 \text { times(s) }\end{array}$ & $\begin{array}{l}40 \text { sets of } \\
\text { data/ the } \\
\text { average } \\
\text { Accuracy c } \\
\text { of20times(\%) }\end{array}$ \\
\hline PSO & 30 & & & & & $\omega=1$ & $1.6,1.5$ & 44.3 & 85.3 \\
\hline WCPSO & 30 & 1 & 0.5 & 6 & 2 & $\omega=0.8$ & Dynamic & 35.5 & 92.6 \\
\hline
\end{tabular}

[16]Propose an improved EMD energy entropy feature vector extraction method, radial basis function (RBF) is used as a support vector machine kernel function parameters, using GA-SVM optimize its punishment factor nuclear parameters and classification functions. Classification accuracy of the GA-SVM method is better than artificial neural network.[17]Proposed based on support vector machines and particle swarm optimization technology (SVM-PSO) method, the application in the field of agriculture, greatly reducing the number of support vector machine, reducing the computational burden. However, this method can not punish a good selection coefficients and kernel function parameters, limiting the performance of SVM.[14]The use of particle swarm optimization algorithm with variable acceleration optimization based on SVM classifier for fault diagnosis of transformer, and achieved good results.[18] According to the characteristics of the mechanical vibration signal high-voltage circuit breakers, put forward a multi-class support vector machine support (MSVM) for fault status classification. Comparative test results show that this method is superior to the wavelet packet analysis (WPT) based on vibration signal analysis, this method failed state classified accurately and quickly. However, this method could not be optimized for the support vector machine, so there is still much to its classification accuracy is room for improvement, adaptive wavelet packet analysis is not, and has its own drawbacks.

Using constant collaboration with dynamic acceleration inertia weight PSO optimized SVM 
classification function parameters $\mathrm{C}$ and kernel function parameters $\sigma$, to overcome the drawbacks of other methods to improve the performance of PSO and SVM classification accuracy.

\section{CONCLUSION}

In this paper, the dynamic acceleration constant collaboration with inertia weight PSO optimized SVM classification function parameters $\mathrm{C}$ and kernel function parameters to improve the performance of the PSO algorithm. IMF component of total energy use as feature vectors characterizing the breaker failure, and as the input feature vectors of SVM, particle swarm algorithm optimized SVM classification, the classification results obtained. Results of comparative experiments show that for high voltage circuit breaker spring mechanism actuator mechanical failure vibration signals, this method can effectively improve the classification accuracy of fault classification and the state, to get a good classification results.

\section{REFERENCES}

[1] Bessedik, S.A. and H. Hadi, Prediction of flashover voltage of insulators using least squares support vector machine with particle swarm optimisation. Electric Power Systems Research, 2013. 104(0): p. 87-92.

[2] Zhao, C., et al., Fault diagnosis of sensor by chaos particle swarm optimization algorithm and support vector machine. Expert Systems with Applications, 2011. 38(8): p. 9908-9912.

[3] $\mathrm{Wu}, \mathrm{Q}$., Fault diagnosis model based on Gaussian support vector classifier machine. Expert Systems with Applications, 2010. 37(9): p 6251-6256.

[4] Tang, X., et al., Multi-fault classification based on support vector machine trained by chaos particle swarm optimization. Knowledge-Based Systems, 2010. 23(5): p. 486-490.

[5] Shi, Z.-b., Q. Song, and M. Ma, Diagnosis for Vibration Fault of Steam Turbine Based on Modified Particle Swarm Optimization Support Vector Machine, in Measuring Technology and Mechatronics Automation Iv, Pts 1 and 2, Z.X. Hou, Editor. 2012. p. 113-116.

[6] Ma, X., Power Transformer Fault Diagnosis Based on Least Squares Support Vector Machine and Particle Swarm Optimization, in Intelligent Structure and Vibration Control, Pts 1 and 2, S.B. Zhong, Y.M. Cheng, and X.L. Qu, Editors. 2011. p. 624-628.

[7] Lee, T.-F., et al., Particle swarm optimization-based SVM application: Power transformers incipient fault syndrome diagnosis. 2006 International Conference on Hybrid Information Technology, Vol 1, Proceedings, ed. G. Lee, et al. 2006. 468-476.

[8] Kennedy J, E.R., Particle swarm optimization. In:Proc.IEEE Int'l.Cof. on Neural Networks,IV.Piscataway,NJ:IEEE Service Center., 1995; 1942-1948.

[9] Guo, H., et al., The Research of Support Vector Machine with Optimized Parameters Based on Particle Swarm Optimization. Istm/2011: 9th International Symposium on Test and Measurement, ed. T.D. Wen. 2011. 96-99.

[10] Fei, S., et al., Application of Particle Swarm Optimization-based Support Vector Machine in Fault Diagnosis of Turbo-generator. 2008 International Symposium on Intelligent Information Technology Application, Vol Ii, Proceedings, ed. Q. Zhou and J. Luo. 2008. 1040-1044.

[11] Cao, J., Fault diagnosis for electrical control system by support vector machine and chaotic particle swarm optimization algorithm. Journal of Computational Information Systems, 2013. 9(12): p. 4931-4938.

[12] Shi, Z.-B., et al. Fault diagnosis of transformer based on quantumbehaved particle swarm optimization-based least squares support vector machines. in 2009 International Conference on Information Engineering and Computer Science, ICIECS 2009,
December 19, 2009 - December 20, 2009. 2009. Wuhan, China: IEEE Computer Society.

[13] Liu, Z., et al., Multi-fault classification based on wavelet SVM with PSO algorithm to analyze vibration signals from rolling element bearings. Neurocomputing, 2013. 99: p. 399-410.

[14] Liao, R.J., et al., A multiclass SVM-based classifier for transformer fault diagnosis using a particle swarm optimizer with time-varying acceleration coefficients. International Transactions on Electrical Energy Systems, 2013. 23(2): p. 181-190.

[15] Emad Elbelttagi, T.H., Donald Grierson, Comparison among five evolution-based optimization algorithms. Advanced Engineering Informations, 2005,19:43-45.

[16] Jian Huang, X.H., Fan Yang, Support vector machine with genetic algorithm for machinery fault diagnosis of high voltage circuit breaker. Measurement, 2011,1018-1027.

[17] ch, S., et al., Optimal design of an in-situ bioremediation system using support vector machine and particle swarm optimization. Journal of Contaminant Hydrology, 2013. 151(0): p. 105-116.

[18] Jian Huang, X.H., Xin Geng, An intelligent fault diagnosis method of high voltage circuit breaker based on improved EMD energy entropy and multi-class support vector machine. Electric Power Systems Research, 2011,400-407. 